\title{
Secular Changes in BMI and Obesity Risk in Japanese Children: Considerations from a Morphologic Perspective
}

\author{
Masaharu Kagawa ${ }^{*}, 1,2$ and Andrew P. Hills ${ }^{1}$ \\ ${ }^{I}$ ATN Centre for Metabolic Fitness, School of Human Movement Studies, Institute of Health and Biomedical Innovation, \\ Queensland University of Technology, Australia \\ ${ }^{2}$ National Institute of Public Health, 2-3-6 Minami, Wako-shi, Saitama, Japan
}

\begin{abstract}
The increasing prevalence of childhood obesity is a global health issue. Past studies in Japan have reported an increase in both body mass index (BMI) and risk of obesity among children and adolescents. However, changes in body size and proportion in this population over time have also influenced BMI. To date, no study of secular changes in childhood obesity has considered the impact of changes in morphological factors. The current study explored the secular changes in BMI and childhood obesity risk among Japanese children from 1950 to 2000 with consideration of changes in body size and the proportions using The Statistical Report of the School Health Survey (SHS). The age of peak velocity (PV) occurred approximately two years earlier in both genders across this period. While the increments in height, sitting height and sub-ischial leg length relative to height levelled off by 1980, weight gain continued in boys. Between 1980 and 2000 , the rate of the upper body weight gain in boys and girls were $0.7-1.3 \mathrm{~kg} / \mathrm{dec} a d e$ and $0.2-1.0 \mathrm{~kg} /$ decade, respectively. After considering body proportions, increments in body weight were small. It could be suggested that the increments in weight and BMI across the 50-year period may be due to a combination of changes including the tempo of growth and body size due to lifestyle factors.
\end{abstract}

Keywords: Secular change, Body proportions, Japan, Children, BMI.

\section{INTRODUCTION}

Obesity is a global health concern and the World Health Organization (WHO) has projected that as many as 2.3 billion individuals above 15 years of age will be overweight and 700 million obese by 2015 [1]. Childhood obesity is an issue of particular concern with an estimated 155 million overweight and between 30 and 45 million obese globally [2].

The body mass index (BMI: $\mathrm{kg} / \mathrm{m}^{2}$ ) is the most commonly utilized index to determine overweight/obesity in both adults and children. Because of its correlation with obesityrelated health conditions, the BMI has been recommended as a screening tool for overweight and obesity in both populations [3-5]. However, as suggested by Garn et al. [6] and later researchers [7], the BMI has major limitations as a screening tool because it is not a measure of adiposity. BMI is 1) height-dependent; 2) proportion-dependent (i.e. leg length relative to height); plus 3 ) the inability to distinguish between fat mass (FM) and fat-free mass (FFM). These limitations suggest that screening ability of BMI will be influenced by physical maturation, race, and gender.

A number of studies have examined secular changes in the BMI of children and concluded that a large proportion of youngsters are overweight or obese [8-11]. However, these studies simply made comparisons between BMI values of

\footnotetext{
*Address correspondence to this author at the Institute of Health and Biomedical Innovation, Queensland University of Technology, 60 Musk Avenue, Kelvin Grove QLD 4059 Australia; Tel: +61-7-3138-6091; Fax:
} +61-7-3138-6030; E-mail: m.kagawa@qut.edu.au groups of the same age in different survey years. These studies did not consider changes in the growth pattern over time, including changes in the timing of puberty and menarche which differ between countries $[12,13]$ and also changes in morphology, including body size and proportions (e.g. leg length relative to height). To date, very few studies have considered adiposity changes, for example using skin fold thickness [14] and no study has reported secular changes in conjunction with body proportions. Since body size and proportions, including leg length, will influence BMI, any interpretation of secular changes without considering these variables may be misleading.

There is a common view that the body size of Japanese children, including height, weight and sub-ischial leg length, has increased since 1950 [15-17]. Many studies have also reported an early onset of puberty $[15,18]$, including menarche in girls [16]. During the same period, other studies have reported an increase in BMI among Japanese children $[19,20]$ and concluded that childhood obesity is increasing in Japan. As many studies did not take changes in growth, body size and proportion into account in recent decades, the actual causes of change in BMI remain unclear. Hermanussen et al. [21] claimed that the increase in BMI among Japanese children and adolescents is due to the earlier onset of puberty and is not indicative of increasing risk of childhood obesity. However, this study did not consider secular change in body proportion and therefore the impact of morphological changes on the secular change in BMI.

The aim of the current study was to examine secular changes in the body size and proportions of Japanese children and adolescents and re-evaluate the secular changes 
in BMI and childhood obesity risk in Japan. The study used the data from The Statistical Report of the School Health Survey (SHS) conducted during the period 1950 to 2000.

\section{MATERIALS AND METHODS}

The SHS is an annual health survey which targets kindergarten and schoolchildren aged between 5 and 17 years. The survey was first conducted in the 1890 s to establish growth standards as part of a growth monitoring system under the auspices of the Japanese Ministry of Education (presently, the Ministry of Education, Science, Culture and Sports; MEXT). The survey is conducted at all schools by law with height, weight and sitting height measured by the school nurse between April and June every year. A randomly extracted sample is used to analyze health status and the growth trends of Japanese schoolchildren. In 2008 , health status was examined using 3,322,441 children and adolescents from 7,755 schools $(22.6 \%$ of the students measured) and growth trends were assessed using 695,600 children ( $4.7 \%$ of the total) [22].

In the present study, gender- and age-specific mean values for 6-17 year-old groups (i.e. elementary school to high school age) for height, weight and sitting height for $1950,1960,1970,1980,1990$, and 2000 were used [23]. The group of 6 year-olds for example, included children aged between 6.00 and 6.99 years. Secular changes in height, weight and sitting height were calculated using the equation: Difference in the variable $=$ [variable in 2000] - [variable in 1950]. Based on these height and weight values, secular change in BMI was also determined. In addition, sub-ischial leg length (height - sitting height) and percentage sub-ischial leg length relative to height (sub-ischial leg length/height $\mathrm{x}$ 100) were calculated in order to observe proportional changes in Japanese children during this period. The age of peak velocity (PV) and PV values for height, weight, sitting height and sub-ischial leg length in each period were determined using the Preece-Baines Model I equation [24]. The equation is appropriate to estimate the age of $\mathrm{PV}$ and is also useful for cross-sectional data analyses $[15,21]$. The estimated values at each age showed very high correlations with the average values above 0.99 for all time-points. Furthermore, the upper body mass was estimated by subtracting a proposed constant proportion of leg mass (16\% per leg) [25] from the weight of children whose leg length relative to the height reached a plateau.

\section{RESULTS}

Secular changes in height and weight in boys and girls during the period 1950-2000 are shown in Tables 1 and 2. There was an $8.1-18.8 \mathrm{~cm}$ or $5.6-13.3 \%$ increase in height and $3.3-15.7 \mathrm{~kg}$ or $17.8-44.1 \%$ increase in weight in boys across the 50-year period. Increments vary between age groups and also according to whether the interpretation is in absolute or relative terms. In boys, the greatest increment in height was observed in the 13-year old group. For weight, the greatest increment in absolute terms was observed in 14year olds whereas using relative values the greatest increment was in the 12-year old group. In contrast, increments in height and weight in girls ranged from 5.4 to $15.4 \mathrm{~cm}(3.5-11.7 \%)$ and 3.4 to $12.4 \mathrm{~kg}(8.1-39.2 \%)$, respectively. Greatest increments for girls were observed in the 11-year old group for height and in the 12-year old group for weight using absolute values (and 11-year olds using the relative value). In summary, both boys and girls showed gains of about $10 \%$ in height and about $40 \%$ in weight in 2000 compared to the children of the same age group in 1950. Compared to girls, boys showed greater increases in body size. Figs. ( 1 and $\mathbf{2}$ ) illustrate pseudo growth curves

Table 1. Height and Weight of Japanese Boys (6 to 17 Years of Age) between 1950 and 2000

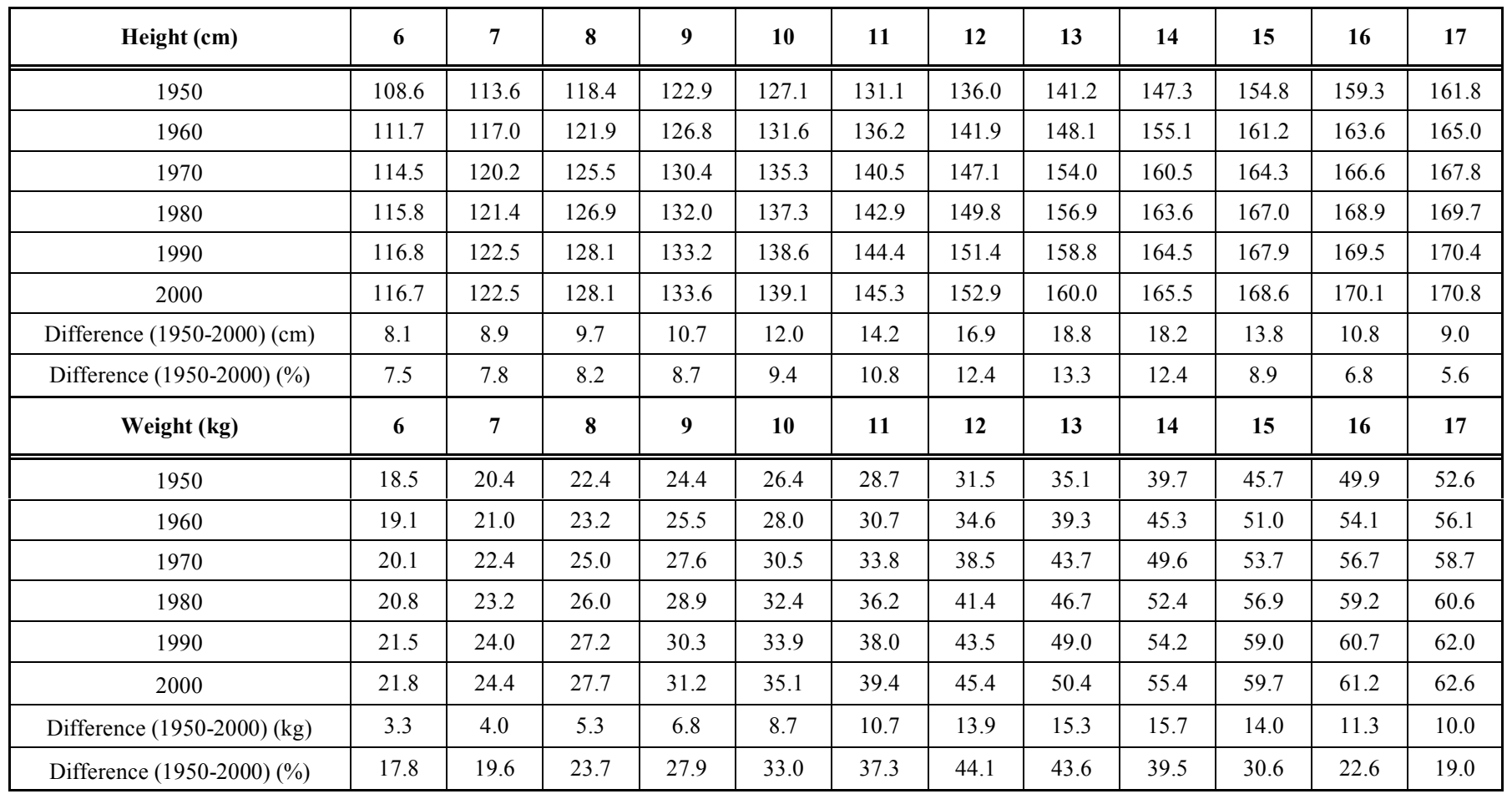


Table 2. Height and Weight of Japanese Girls (6 to 17 Years of Age) between 1950 and 2000

\begin{tabular}{|c|c|c|c|c|c|c|c|c|c|c|c|c|}
\hline Height (cm) & 6 & 7 & 8 & 9 & 10 & 11 & 12 & 13 & 14 & 15 & 16 & 17 \\
\hline 1960 & 110.6 & 115.9 & 121.1 & 126.3 & 132.0 & 138.1 & 144.0 & 148.1 & 150.7 & 152.7 & 153.3 & 153.7 \\
\hline 1980 & 114.9 & 120.6 & 126.2 & 131.9 & 138.3 & 144.9 & 150.6 & 154.0 & 156.0 & 156.6 & 156.9 & 157.0 \\
\hline 1990 & 116.0 & 121.8 & 127.4 & 133.1 & 139.5 & 146.3 & 151.5 & 154.7 & 156.4 & 157.2 & 157.6 & 157.9 \\
\hline Difference $(1950-2000)(\mathrm{cm})$ & 8.0 & 8.9 & 9.9 & 11.4 & 13.7 & 15.4 & 14.8 & 12.6 & 10.2 & 7.1 & 5.9 & 5.4 \\
\hline Difference (1950-2000) (\%) & 7.4 & 7.9 & 8.4 & 9.3 & 10.8 & 11.7 & 10.8 & 8.8 & 7.0 & 4.7 & 3.9 & 3.5 \\
\hline Weight (kg) & 6 & 7 & 8 & 9 & 10 & 11 & 12 & 13 & 14 & 15 & 16 & 17 \\
\hline 1950 & 17.9 & 19.8 & 21.8 & 23.8 & 26.0 & 28.8 & 32.6 & 36.9 & 41.2 & 45.2 & 47.7 & 49.1 \\
\hline 1990 & 21.1 & 23.6 & 26.6 & 29.9 & 34.0 & 38.9 & 43.9 & 47.5 & 50.2 & 52.1 & 52.6 & 52.8 \\
\hline 2000 & 21.3 & 23.8 & 27.0 & 30.7 & 34.9 & 40.1 & 45.0 & 48.3 & 50.7 & 52.1 & 53.0 & 53.1 \\
\hline Difference (1950-2000) (kg) & 3.4 & 4.0 & 5.2 & 6.9 & 8.9 & 11.3 & 12.4 & 11.4 & 9.5 & 6.9 & 5.3 & 4.0 \\
\hline Difference (1950-2000) (\%) & 19.0 & 20.2 & 23.9 & 29.0 & 34.2 & 39.2 & 38.0 & 30.9 & 23.1 & 15.3 & 11.1 & 8.1 \\
\hline
\end{tabular}

a) Boys

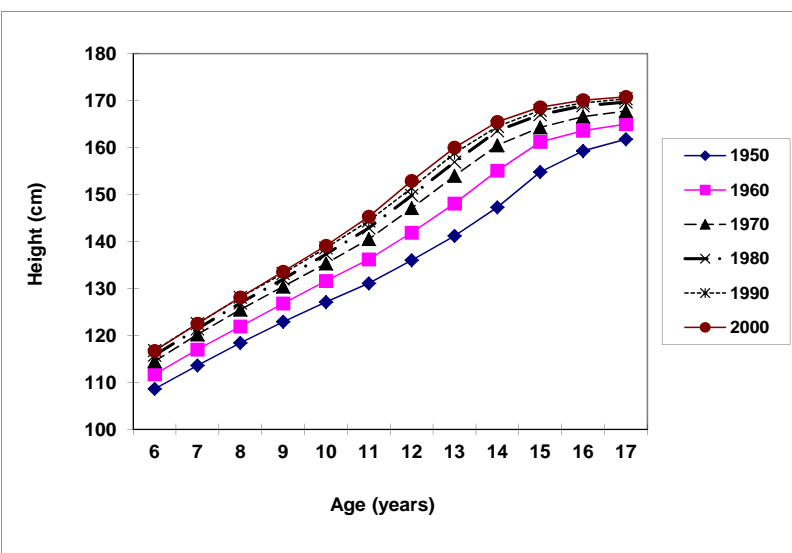

b) Girls

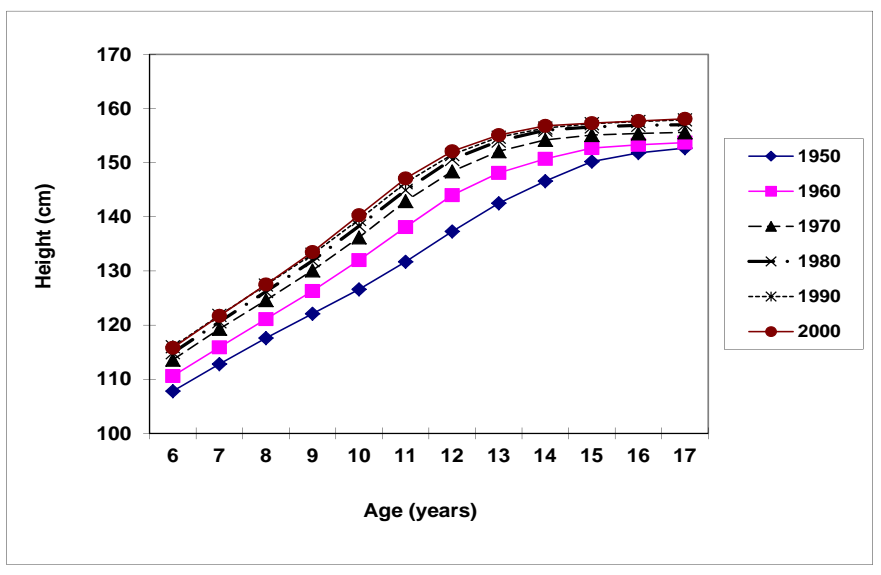

Fig. (1). Comparison of pseudo growth curves for height for a) boys and b) girls between 1950 and 2000 .

a) Boys

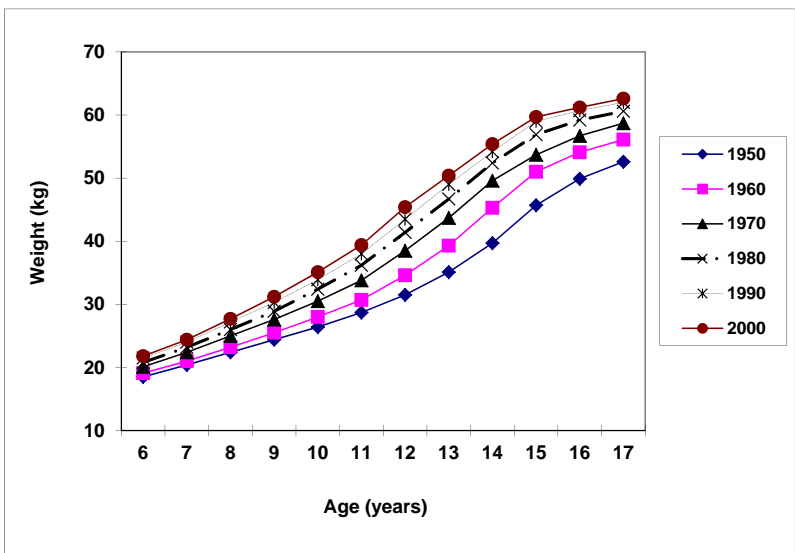

b) Girls

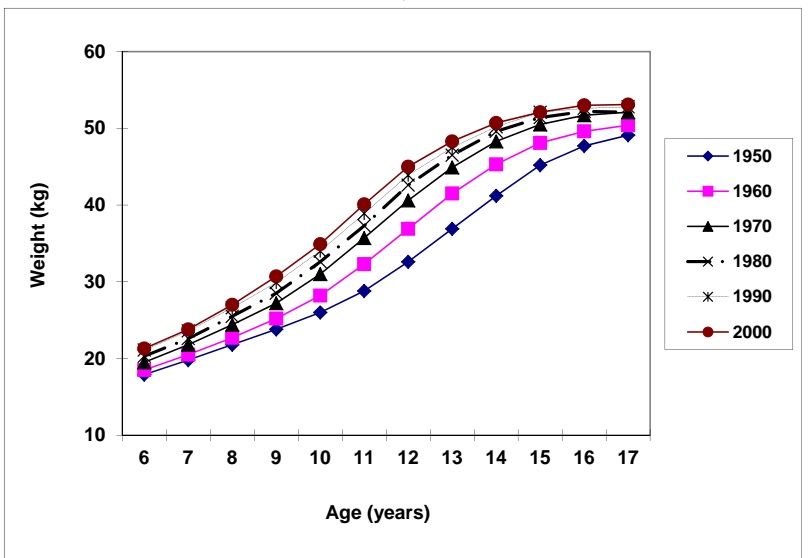

Fig. (2). Comparison of pseudo growth curves for weight for a) boys and b) girls between 1950 and 2000. 
a) Boys

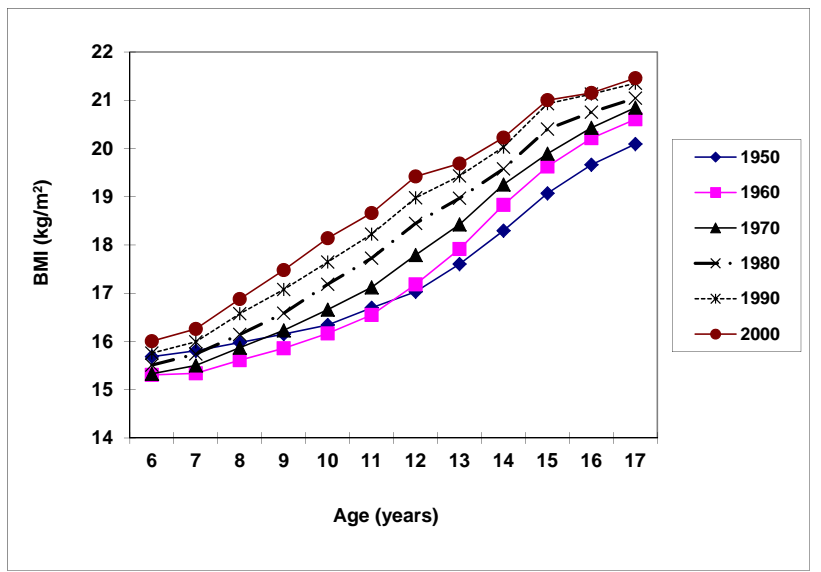

b) Girls

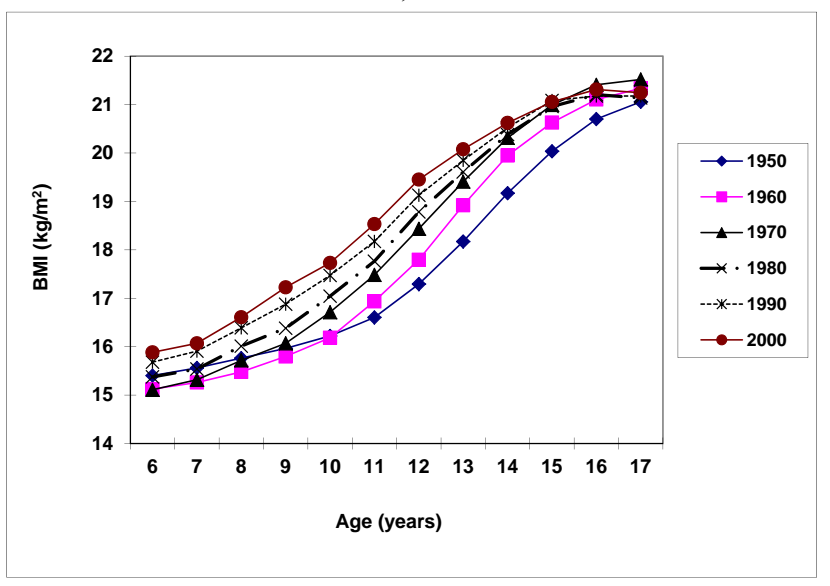

Fig. (3). Comparison of pseudo growth curves for BMI for a) boys and b) girls between 1950 and 2000

for height and weight. All figures clearly show a rapid increment between 1950 and 1960 and then the rate of increment starts to decelerate. While height for both genders and weight for girls overlapped between 1980 and 2000, weight for boys appeared to increase continuously from 1980 to 1990 and then become steady.

Differences in the patterns of height and weight between the time points and genders were also reflected in secular changes in BMI (Fig. 3). Compared to the BMI curve in 1950, the 1960 curve for boys showed a lower BMI until 12 years of age followed by a rapid increase afterwards. Similarly, the curve for 1970 revealed a lower BMI until 9 years of age then higher BMI afterwards. In 1980, the BMI of the 6-year old group was comparable with that of 1950 but then BMI increased after 1970 in all age groups. In addi- tion, girls showed another distinguishable pattern compared to boys. BMI values for girls in the 17-year age group were consistent over the last 50 years suggesting that girls have maintained a balance between height and weight while weight relative to height in boys has been increasing.

Table 3 shows that the age of PV calculated for height, weight, sitting height and sub-ischial leg length over the examined period declined by approximately two years in both genders. The decline in PV was greatest between 1950 and 1960. While PV was first observed in sub-ischial leg length followed by height for both genders, boys experienced PV for weight and sitting height at the same time whereas girls experienced PV for sitting height prior to weight.

Table 3. Differences in Age of Peak Velocity for Height, Weight, Sitting Height and Sub-Ischial Leg Length in Japanese Boys and Girls between 1950 and 2000

\begin{tabular}{|c|c|c|c|c|c|c|c|c|}
\hline \multirow{2}{*}{ Year } & \multicolumn{2}{|c|}{ Height (cm) } & \multicolumn{2}{|c|}{ Weight (kg) } & \multicolumn{2}{|c|}{ Sitting height $(\mathrm{cm})$} & \multicolumn{2}{|c|}{ Sub-ischial leg length $(\mathrm{cm})$} \\
\hline & Age of PHV & PHV & Age of PWV & PWV & Age of PHV & PHV & Age of PV & PV \\
\hline \multicolumn{9}{|c|}{ Boys } \\
\hline 1950 & 14.1 & 7.02 & 14.4 & 5.57 & 14.4 & 3.76 & 13.6 & 3.39 \\
\hline 1960 & 13.2 & 7.17 & 13.7 & 6.04 & 13.7 & 3.92 & 12.7 & 3.43 \\
\hline 1970 & 12.6 & 7.09 & 13.1 & 5.63 & 13.1 & 3.67 & 12.1 & 3.61 \\
\hline 1990 & 12.2 & 7.39 & 12.6 & 5.89 & 12.7 & 3.72 & 11.8 & 3.83 \\
\hline 2000 & 12.0 & 7.54 & 12.3 & 5.69 & 12.4 & 3.68 & 11.6 & 3.99 \\
\hline \multicolumn{9}{|c|}{ Girls } \\
\hline 1950 & 12.0 & 5.45 & 13.2 & 4.60 & 12.6 & 2.80 & 11.3 & 2.81 \\
\hline 1980 & 10.3 & 6.71 & 11.3 & 5.16 & 10.8 & 3.42 & 9.8 & 3.45 \\
\hline 1990 & 10.1 & 6.62 & 11.0 & 4.99 & 10.5 & 3.30 & 9.7 & 3.41 \\
\hline 2000 & 9.9 & 6.85 & 10.7 & 5.04 & 10.2 & 3.36 & 9.6 & 3.54 \\
\hline
\end{tabular}


Table 4. Differences in the Upper Body Mass between 1960 and 2000 for 11-17 Years Old Japanese Boys and Girls

\begin{tabular}{|c|c|c|c|c|c|c|c|c|}
\hline & Age & 11 & 12 & 13 & 14 & 15 & 16 & 17 \\
\hline \multirow{7}{*}{ Boys } & Difference (1960-1970) (kg) & 2.1 & 2.7 & 3.0 & 2.9 & 1.8 & 1.8 & 1.8 \\
\hline & Difference (1980-1990) (kg) & 1.2 & 1.4 & 1.6 & 1.2 & 1.4 & 1.0 & 1.0 \\
\hline & Difference (1990-2000) (kg) & 1.0 & 1.3 & 1.0 & 0.8 & 0.5 & 0.3 & 0.4 \\
\hline & $\%$ increment of upper body mass $(1960-2000)$ & 28.3 & 31.2 & 28.2 & 22.3 & 17.1 & 13.1 & 11.6 \\
\hline & Rate of increment $(\mathrm{kg})$ per decade $(1960-2000)$ & 1.5 & 1.8 & 1.9 & 1.7 & 1.5 & 1.2 & 1.1 \\
\hline & $\%$ increment of upper body mass $(1980-2000)$ & 8.8 & 9.7 & 7.9 & 5.7 & 4.9 & 3.4 & 3.3 \\
\hline & Rate of increment $(\mathrm{kg})$ per decade $(1980-2000)$ & 1.1 & 1.4 & 1.3 & 1.0 & 1.0 & 0.7 & 0.7 \\
\hline \multirow{6}{*}{ Girls } & Difference (1990-2000) (kg) & 0.8 & 0.7 & 0.5 & 0.3 & 0.0 & 0.3 & 0.2 \\
\hline & Difference (1960-2000) (kg) & 5.3 & 5.5 & 4.6 & 3.7 & 2.7 & 2.3 & 1.8 \\
\hline & $\%$ increment of upper body mass $(1960-2000)$ & 24.1 & 22.0 & 16.4 & 11.9 & 8.3 & 6.9 & 5.4 \\
\hline & Rate of increment $(\mathrm{kg})$ per decade $(1960-2000)$ & 1.3 & 1.4 & 1.2 & 0.9 & 0.7 & 0.6 & 0.5 \\
\hline & $\%$ increment of upper body mass $(1980-2000)$ & 7.5 & 5.6 & 3.9 & 2.2 & 1.4 & 1.5 & 1.9 \\
\hline & Rate of increment $(\mathrm{kg})$ per decade $(1980-2000)$ & 1.0 & 0.8 & 0.6 & 0.4 & 0.2 & 0.3 & 0.3 \\
\hline
\end{tabular}

Note: 11 and 12 years old boys have not reached optimum proportion of sub-ischial leg length relative to height yet and therefore the proportion of leg mass relative to the total body weight may not be equivalent to other age groups.

Fig. (4) illustrates the change in proportion of sub-ischial leg length relative to height with a continuous increase from 1950 to 1980 in both genders. The proportion is consistent after 1980 which indicates that the Japanese reached the optimum proportion of sub-ischial leg length relative to height by 1980 . The change in proportion reached a plateau about 13 years of age for boys and about 11 years of age for girls across the survey periods except for 1950 where the peak was observed approximately one year later.

Table 4 shows changes in the upper body mass of Japanese children based on a constant proportion of leg mass relative to weight from 1960 to 2000 . Using the ages 13 years and above, boys gained an upper body mass of 4.4-7.5 $\mathrm{kg}$ whereas girls aged above 11 years old gained $1.8-5.5 \mathrm{~kg}$. The magnitude of the upper body mass gain depends on the age group with increments from 1.1-1.9 kg/decade for boys and $0.5-1.4 \mathrm{~kg} / \mathrm{decade}$ for girls. In addition, a comparison of the upper body mass gain during the period 1980 to 2000 (where the proportion of sub-ischial leg length was consistent) there were minimal increments, $0.7-1.3 \mathrm{~kg} /$ decade for boys and $0.2-1.0 \mathrm{~kg} / \mathrm{decade}$ for girls.

\section{a) Boys}

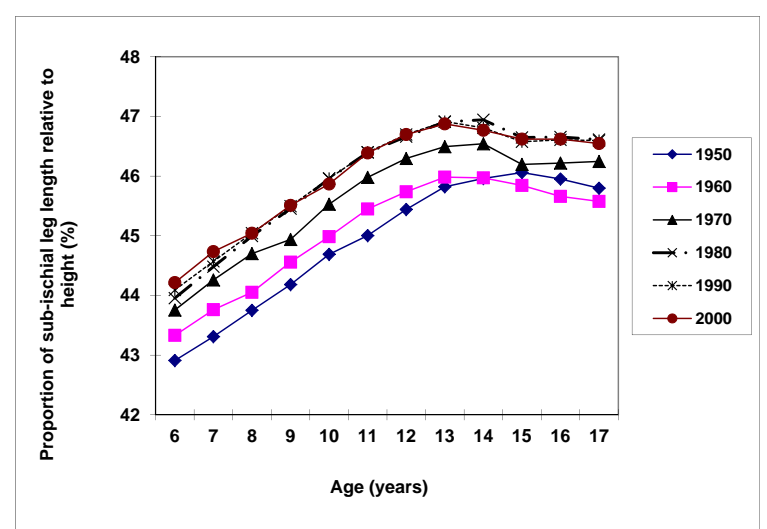

b) Girls

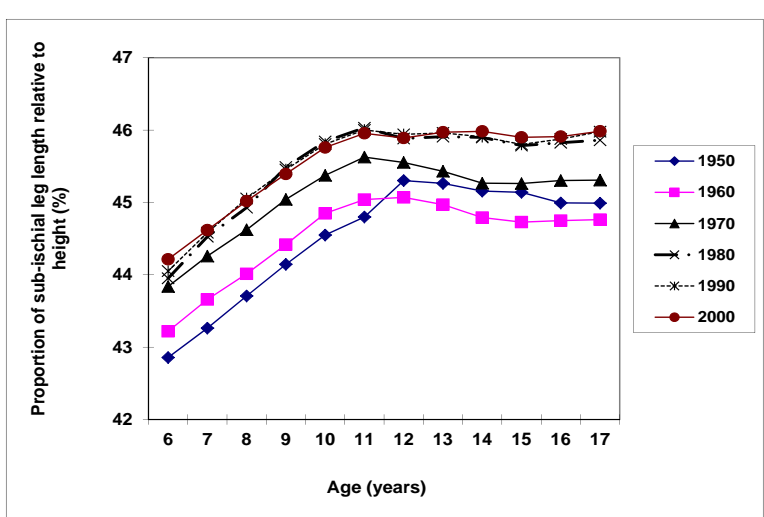

Fig. (4). Comparison of pseudo growth curves for proportion of sub-ischial leg length relative to height for a) boys and b) girls between 1950 and 2000 . 


\section{DISCUSSION}

Increments in height and weight over the 50-year period in both genders confirm previous reports of rapid growth during the post-World War II period [15, 16, 18]. The increments in height and weight from 1950 to 1960 are rapid compared to the rest of the survey period which can be explained by acceleration in the tempo of growth. The acceleration in the onset of PV in Japanese children is consistent with a previous study [21] in which Japanese children showed a decrease in the age of onset of PV by about two years. However, the current study revealed that the age of PV for all assessed variables decreased rapidly for about a year during the period of 1950 to 1960 . Such a dramatic change in body size may reflect the recovery of many Japanese children from a malnourished state during World War II [26, 27] as Japan experienced rapid economic growth [28].

In the current study, children under the age of 9 years in 1960 and 1970 showed smaller BMI values than children of the same age in 1950. This suggests a greater height increment relative to weight gain during this period. This may be associated with increased energy intake from fat [27] but more importantly, increased intake of milk and dairy products which may be attributed to the school lunch program resumed in a complete form (inclusion of bread, milk and a side dish) from 1950 [29]. Therefore it can be suggested that this greater height increment relative to weight gain by young Japanese children during this period was a result of improved nutritional status, including increased calcium intake, which resulted in the change in the timing of puberty. Except for children under 9 years of age in 1960 and 1970, Japanese children experienced a considerable increase in BMI compared to 1950 . This reflects a greater weight gain relative to height increment. Weight gain continued until 2000 but height reached a steady rate in both genders by 1980.

In addition to height and weight, body proportions as illustrated by the sub-ischial leg length relative to height continued to increase until 1980 and reached a plateau. This suggests that Japanese children reached a genetically optimum height and proportionality consistent with desirable environmental factors including adequate nutrient and energy intakes by 1980 . The observed results conflict with an earlier suggestion that Japanese reached their maximum physical size by 1970 [27]. The difference may be attributed to study design. The present study reported on growth patterns based on changes in the age of onset of PV and the actual pseudo growth curves of the measured variables. In contrast, the findings of the previous study were only based on the magnitude of decline in age of onset for PHV and difference in height of the 17-year old groups for the survey years.

The increase in BMI seen in the current study is consistent with the global trend across the second half of the 20th century [9-11]. An earlier study of 6-12 year-old Japanese children measured in 1989 and also in 2001/02 reported a rapid increase of children with a BMI above the age- and gender-specific 95th percentile cut-off point [20]. To date, however, no studies have considered changes in body size and proportion when interpreting the secular change of the BMI in Japanese children. In the period from 1950 to 1980 ,
Japanese children experienced an increase in height, which is partly due to an increase in leg length as well as increase in the proportion of leg length relative to height as reported in previous studies $[15,17]$. While BMI decreases as the proportion of leg length relative to height increases, increases in height alone will commonly lead to increases in weight due to gains in FM and FFM. Therefore, an increase in BMI during this period may be partly due to the combined effect of accelerated tempo and changes in body size rather than deposition of excessive body fat alone. Since BMI cannot provide any details regarding body composition, it is impossible to determine the actual cause of weight gain during this period. This complicates both the interpretation of BMI and secular change in obesity risk among Japanese children during this period.

Based on adult data, the proportion of mass attributed to the lower limb is constant (about $16 \%$ per leg) regardless of race and gender [25]. Assuming that children have a comparable body proportion to adults at the age they reach peak sub-ischial leg length relative to height, we calculated the upper body mass to gain a better indication of trunk fat accumulation. Since 1960, both genders showed a comparable tempo of growth between survey years. During the same period, boys and girls experienced an upper body mass gain of $4.4-7.5 \mathrm{~kg}$ (1.1-1.9 kg/decade) and $1.8-5.5 \mathrm{~kg}(0.5-1.4$ $\mathrm{kg} / \mathrm{decade}$ ), respectively. The curves representing the proportion of sub-ischial leg length relative to height become identical from 1980. As height was also consistent after 1980 , increases in weight and BMI after this time point may be attributed to lifestyle factors including energy intake and physical activity. However, the results showed minimal weight gain in the upper body region in both genders. The findings of the current study conflict with those of a previous study which reported a rapid increase in childhood obesity [20]. However, the previous study focused on 6-12 year-old children whereas the current study considered data from 1117 year-old children whose proportion of sub-ischial leg length relative to height reached a plateau. However, we observed a greater difference in BMI in young children between 1980 and 2000 compared with adolescents (Fig. 3) and also a greater \% increment in the upper body mass in younger age groups (Table 4). These observations may suggest variability in obesity risk between age groups with younger children being more vulnerable to weight gain in the upper body, which may have implications for the development of metabolic complications. However, this possibility needs to be clarified in future research as the assumption of a constant proportion of mass from the legs across childhood may not hold given the differences in proportionality between children and adults.

In comparison to boys, girls aged between 15 and 17 years showed comparable BMI values since 1960. Previous studies have not discussed such gender differences during the period where the tempo of growth and body proportion are consistent. One of the possible reasons may be an increased prevalence of the 'ideal' thin female body by Japanese adolescent girls. Results from previous studies suggest that girls, particularly those living in metropolitan areas, tend not to become obese compared to boys or girls living in rural areas [19] and that there has been an increase in the proportion of young females whose BMI is below 18.5 $\mathrm{kg} / \mathrm{m}^{2}[30]$. 
The small weight gains in Japanese children reported in this study do not mean they are without risk from obesity. A previous study with adults suggested that the proportion of weight gain from FM varies between individuals depending on the amount of FM they have at the time of weight gain [31]. It has also been reported that an increasing proportion of Japanese children are sedentary [27] which may place them at increased risk of weight gain from FM in the future. To prevent this, effective obesity prevention strategies are required.

A limitation of the current study was the use of adult values to calculate the proportion of leg mass relative to total weight $(32 \%)$ [25]. Since body proportion in children alters during growth there may also be a difference in the proportion of leg mass relative to total weight between children and adults. Previous studies that examined body composition of children under 14 years of age using DXA reported the proportion of leg mass as approximately $35 \%$ regardless of gender and obesity status $[32,33]$. In order to clarify the wider applicability of the adult values and to identify the impact of tempo and change in body proportions, it is recommended that age- and gender-specific proportions of leg mass relative to height to be determined in future studies.

\section{CONCLUSION}

To our knowledge, no previous study has considered increases in BMI in conjunction with changes in body size and proportion. After accounting for these factors, the current study found that increments in body weight were small and the subsequent risk of childhood obesity may not be increasing as rapidly as it has been suggested among Japanese children. Results suggest that increases in weight and BMI may be the result of both changes in tempo as well as body size due to lifestyle factors. Considering the potential for higher body weight in younger age groups and the increasingly sedentary lifestyle of many Japanese, preventative strategies are recommended to minimise childhood obesity and related metabolic complications.

\section{REFERENCES}

[1] WHO. Obesity and overweight; 2006 September. Report No.: 311.

[2] Lobstein $\mathrm{T}$, Baur L, Uauy R, IASO International Obesity Taskforce. Obesity in children and young people: a crisis in public health. Obes Rev 2004; 5: 4-85.

[3] WHO. Obesity: Preventing and managing the global epidemic. Geneva: WHO/NUT/NCD/98.1; 1997.

[4] Cole TJ, Bellizzi MC, Flegal KM, Dietz WH. Establishing a standard definition for child overweight and obesity worldwide: international survey. Br Med J 2000; 320: 1240-5.

[5] WHO expert consultation. Appropriate body-mass index for Asian populations and its implications for policy and intervention strategies. Lancet 2004; 363: 157-63.

[6] Garn SM, Leonard WR, Hawthorne VM. Three limitations of the body mass index. Am J Clin Nutr 1986; 44: 996-7.

[7] Deurenberg P, Deurenberg-Yap M, Guricci S. Asians are different from Caucasians and from each other in their body mass index/body fat per cent relationship. Obes Rev 2002; 3: 141-6.

[8] Magarey AM, Daniels LA, Boulton TJ. Prevalence of overweight and obesity in Australian children and adolescents: reassessment of 1985 and 1995 data against new standard international definitions. Med J Aust 2001; 174: 561-4.
[9] Chrzanowska M, Koziel S, Ulijaszek SJ. Changes in BMI and the prevalence of overweight and obesity in children and adolescents in Cracow, Poland, 1971-2000. Econ Hum Biol 2007; 5: 370-8.

[10] Ji CY, Chen TJ. Secular changes in stature and body mass index for Chinese youth in sixteen major cities, 1950s-2005. Am J Hum Biol 2008; 20: 530-7.

[11] Zhang YX, Wang SR. Distribution of body mass index and the prevalence changes of overweight and obesity among adolescents in Shandong, China from 1985 to 2005. Ann Hum Biol 2008; 35: 547-55.

[12] Euling SY, Selevan SG, Pescovitz OH, Skakkebaek NE. Role of environmental factors in the timing of puberty. Pediatrics 2008; 121: S167-S71.

[13] Euling SY, Herman-Giddens ME, Lee PA, et al. Examination of US puberty-timing data from 1940 to 1994 for secular trends: panel findings. Pediatrics 2008; 121: S172-S91.

[14] Mészáros Z, Mészáros J, Völgyi E, et al. Body mass and body fat in Hungarian schoolboys: differences between 1980-2005. J Physiol Anthropol Appl Human Sci 2008; 27: 241-5.

[15] Tanner JM, Hayashi T, Preece MA, Cameron N. Increase in length of leg relative to trunk in Japanese children and adults from 1957 to 1977: comparison with British and with Japanese Americans. Ann Hum Biol 1982; 9: 411-23.

[16] Tsuzaki S, Matsuo N, Ogata T, Osano M. Lack of linkage between height and weight and age at menarche during the secular shift in growth of Japanese children. Ann Hum Biol 1989; 16: 429-36.

[17] Ali MA, Uetake T, Ohtsuki F. Secular changes in relative leg length in post-war Japan. Am J Hum Biol 2000; 12: 405-16.

[18] Matsumoto K. Secular acceleration of growth in height in Japanese and its social background. Ann Hum Biol 1982; 9: 399-410.

[19] Matsushita Y, Yoshiike N, Kaneda F, Yoshita K, Takimoto H. Trends in childhood obesity in Japan over the last 25 years from the national nutrition survey. Obes Res 2004; 12: 205-14.

[20] Yoshinaga M, Shimago A, Koriyama C, et al. Rapid increase in the prevalence of obesity in elementary school children. Int J Obes 2004; 28: 494-9.

[21] Hermanussen M, Molinari L, Satake T. BMI in Japanese children since 1948: no evidence of a major rise in the prevalence of obesity in Japan. Anthropol Anz 2007; 65: 275-83.

[22] Ministry of Education Science Culture and Sports (MEXT). Announcement on 2008 School Health Statistical Survey [Jap] 2009 [cited 2009 9/1]; Available from: http://www.mext.go.jp/ b_menu/toukei/001/h20.htm

[23] Ministry of Education Culture Sports Science and Technology (MEXT). The Statistical Report of the School Health Survey. 2009 [cited 2009 14/09]; Available from: http://www.mext.go.jp/ b_menu/toukei/001/index03.htm

[24] Preece MA, Baines MJ. A new family of mathematical models describing the human growth curve. Ann Hum Biol 1978; 5: 1-24.

[25] Osterkamp LK. Current perspective on assessment of human body proportions of relevance to amputees. J Am Diet Assoc 1995; 95: 215-8.

[26] Kimura K. Studies on growth and development in Japan. Yearbook Phys Anthropol 1984; 27: 179-214.

[27] Murata M. Secular trends in growth and changes in eating patterns of Japanese children. Am J Clin Nutr 2000; 72: 1379S-83S.

[28] Statistics Bureau Director-General for Policy Planning \& Statistical Research and Training Institute. System of National Accounts (68SNA). [cited 2009; Available from: http://www.stat.go.jp/data/ chouki/03.htm

[29] Ministry of Education Culture Sports Science and Technology (MEXT). Promotion of the school lunch program [Jap]. [cited 2008 18/04/2009]; Available from: http://www.mext.go.jp/b_menu/ hakusho/html/hpbz198101/hpbz198101_2_185.html

[30] Takimoto H, Yoshiike N, Kaneda F, Yoshita K. Thinness among young Japanese women. Am J Public Health 2004; 94: 1592-5.

[31] Forbes GB. Body fat content influences the body composition response to nutrition and exercise. Ann NY Acad Sci 2000; 904: 359-65.

[32] Fuller NJ, Fewtrell MS, Dewit O, Elia M, Wells JC. Segmental bioelectrical impedance analysis in children aged 8-12 y: 2 . The assessment of regional body composition and muscle mass. Int $\mathrm{J}$ Obes 2002; 26: 692-700. 
[33] Wells JC, Fewtrell MS, Williams JE, Haroun D, Lawson MS, Cole TJ. Body composition in normal weight, overweight and obese children: matched case-control analyses of total and regional tissue masses, and body composition trends in relation to relative weight. Int J Obes 2006; 30: 1506-13.

(C) Kagawa and Hills; Licensee Bentham Open.

This is an open access article licensed under the terms of the Creative Commons Attribution Non-Commercial License (http: //creativecommons.org/licenses/by$\mathrm{nc} / 3.0 /$ ), which permits unrestricted, non-commercial use, distribution and reproduction in any medium, provided the work is properly cited. 